\title{
Antineutrophil antibody: a test for autoimmune primary sclerosing cholangitis in childhood?
}

\author{
S K Lo, R W G Chapman, P Cheeseman, C P J Charlton, J A Walker-Smith, G Mieli-Vergani, \\ K A Fleming
}

\begin{abstract}
The detection of an antineutrophil antibody which is highly sensitive and specific for adult primary sclerosing cholangitis using indirect immunoalkaline phosphatase has been previously described. In this study, the diagnostic potential of this method in childhood primary sclerosing cholangitis is described. A range of 72 blinded children's sera ( 36 boys), aged six months to 21 years (10 primary sclerosing cholangitis, eight autoimmune chronic active hepatitis, 10 alpha-1 antitrypsin deficiency, 12 extrahepatic bile duct atresia, 11 ulcerative colitis and 21 normal subjects) was assayed. Eight of the 10 primary sclerosing cholangitis patients were correctly identified. Three patients with chronic active hepatitis also showed the characteristic primary sclerosing cholangitis pattern of staining. No ulcerative colitis patients or any other patients showed this pattern of staining. All normal subjects were negative. As in adult primary sclerosing cholangitis, there is a specific antineutrophil antibody in childhood primary sclerosing cholangitis and this provides further evidence towards an autoimmune aetiology of this condition. The test may have diagnostic potential.
\end{abstract}

(Gut 1993; 34: 199-202)

Department of Gastroenterology, John Radcliffe Hospital, Oxford

$S$ K Lo

R W G Chapman

Department of Child

Health, King's College

School of Medicine and

Dentistry, King's

College, London

P Cheeseman

G Mieli-Vergani

Academic Department of Paediatric

Gastroenterology, Queen

Elizabeth Hospital for

Children, London

C P J Charlton

J A Walker-Smith

Nuffield Department of Pathology and

Bacteriology, University of Oxford, John Radcliffe

Hospital, Oxford

K A Fleming

Correspondence to:

Dr K A Fleming, Nuffield

Department of Pathology and

Bacteriology, University of

Oxford, John Radcliffe

Hospital, Oxford OX3 9DU.

Accepted for publication

14 July 1992
Primary sclerosing cholangitis is a chronic cholestatic liver disease characterised by an inflammatory fibrosis of both the intra and extrahepatic bile ducts. Seventy per cent of the patients have an associated inflammatory bowel disease especially ulcerative colitis. ${ }^{12}$ The disease is being diagnosed with increasing frequency among adult patients with the advent of endoscopic retrograde cholangiography. This technique remains the gold standard in diagnosing primary sclerosing cholangitis. Primary sclerosing cholangitis has been described very rarely in childhood and this may be attributed to the limited use of endoscopic retrograde cholangiography in children. Before 1987, only five children with childhood primary sclerosing cholangitis were reported in the literature. ${ }^{3-5}$ Then, a series of 13 children with primary sclerosing cholangitis was described from King's College Hospital, London, suggesting a higher prevalence of primary sclerosing cholangitis in childhood than previously suspected. ${ }^{6}$

We have previously reported a method of detecting a highly specific and sensitive antineutrophil antibody in adult primary sclerosing cholangitis. ${ }^{7}$ The aim of this study is to determine the diagnostic potential of this method in childhood primary sclerosing cholangitis, especially given the highly invasive nature of endoscopic retrograde cholangiography.

\section{Methods}

PATIENT POPULATION AND SERUM SAMPLES

The project was approved by the relevant local ethical committees.

\section{PRIMARY SCLEROSING CHOLANGITIS}

The diagnosis of primary sclerosing cholangitis was based on clinical, biochemical, histological, and endoscopic retrograde cholangiography criteria. Five of the 10 children were boys. Their mean age at the time of blood sampling was $11 \cdot 7$ years (range 6-20). Only one girl had symptoms of chronic liver disease (tiredness, anorexia, and right upper abdominal pain). Three children had ulcerative colitis and four had undetermined colitis. All children who had colitis were asymptomatic except one boy who had a bowel frequency of up to six times per day. There were three girls who had no associated inflammatory bowel disease. Nine children were on prednisolone. In addition, five patients were receiving azathioprine and one patient was also given colchicine and ursodeoxycholic acid. The liver histology and biochemistry were as in Tables I and II. All patients underwent endoscopic retrograde cholangiography and all had ductal changes consistent with primary sclerosing cholangitis.

\section{OTHER CHILDHOOD DISEASES}

The study also included 10 children with alpha-1 anti-trypsin deficiency (PIZZ phenotype, age range $8-20$ years, mean $12 \cdot 3$, three boys), 12 with extrahepatic bile duct atresia (age range 7-13 years, mean $10 \cdot 7$, five boys) and eight chronic active hepatitis (age range 7-21 years, mean $14 \cdot 2$, four boys). Four of the last group of patients have Type 1 chronic active hepatitis (positive antismooth muscle and nuclear antibodies) and the other four children have Type 2 chronic active hepatitis (positive antiliver-kidney microsomal antibody). All the diagnosis were made on conventional criteria.

\section{ULCERATIVE COLITIS}

A total of 11 children with ulcerative colitis was also studied. The diagnosis was made on clinical, colonoscopic and histological criteria. The mean age of this group was $13 \cdot 1$ years (range 9-19). Six patients were boys. The mean duration of the disease was five years (range 2-10). Eight patients were in remission, two had active colitis and one 
TABLE I Liver histology of the primary sclerosing cholangitis patients

\begin{tabular}{ll}
\hline Stage & $(n)$ \\
\hline 0 & 0 \\
1 & 2 \\
2 & 3 \\
3 & 3 \\
4 & 2 \\
\hline
\end{tabular}

patient had had previous colectomy. None of the patients had stigmata of chronic liver disease or hepatomegaly. All their biochemical markers of liver disease (alkaline phosphatase and aspartate aminotransferase) were normal.

\section{NORMAL CONTROLS}

Normal controls were recruited through the Medway and Gillingham branch of the Children's Liver Disease Foundation. Blood was taken after obtaining informed consent from parents or from the older children. None of these subjects had chronic diarrhoea or any significant past medical history. All had normal liver biochemical tests. Twelve of the total 21 children were male. The mean age was 5.9 years (range six months 11 years).

The sera were tested totally blinded. All sera were stored at $-20^{\circ} \mathrm{C}$ and thawed slowly at room temperature just before use.

\section{ISOLATION OF NEUTROPHILS}

Normal peripheral blood from a single healthy adult was used in the study. Neutrophils were separated by centrifuging blood with JPM Prep (TechGen International, London) at $500 \mathrm{~g}$ for 30 minutes. Harvested neutrophils were washed with $5 \%$ fetal calf serum (Tissue Culture Service) in Tris buffered saline $(50 \mathrm{mM}$ Tris, $145 \mathrm{mM}$ $\mathrm{NaCl}, \mathrm{pH} 7 \cdot 6$ ) to remove JPM Prep followed by a further five minute centrifugation of $500 \mathrm{~g}$ to precipitate the cells. The cell pellet was resuspended in the same buffer. Aliquots of the cells were cytocentrifuged (Shandon Southern Cytocentrifuge, Cheshire, UK) at $500 \mathrm{rpm}$ for five minutes onto slides. About 75000 neutrophils per slide were prepared. These were air dried for 30 minutes and then fixed with absolute alcohol for five minutes. Fixed cells were incubated with coded patients' sera diluted 1:10 in Tris buffered saline for 30 minutes. After washing with Tris buffered saline for one minute, bound antibody was detected using indirect immunohistochemistry. Rabbit antibody to human immunoglobulin heavy chains conjugated with alkaline phosphatase (Dako, High Wycombe, UK) at a dilution of 1:30 in Tris buffered saline was used as secondary antibody. After 30 minute incubation with the antibody, the cells were washed in Tris buffered saline. Slides were incubated in Fast Red TR salt (Sigma) as substrate for 12 minutes. Known positive adult primary sclerosing cholangitis and negative normal sera were used as appropriate controls. All procedures were carried out at room temperature. Slides were examined independently by two observers (SKL, KAF) using light microscopy.

TABLE II Liver biochemistry of the primary sclerosing cholangitis patients

\begin{tabular}{lcc}
\hline & Mean & Range \\
\hline Bilirubin normal: 3-20 $\mu \mathrm{mol} / \mathrm{l}$ & $18 \cdot 44$ & $4-51$ \\
Alkaline phosphatase normal: 30-85 IU/1 & $289 \cdot 3$ & $17-553$ \\
Aspartate aminotransferase normal: & & \\
$\begin{array}{l}\text { 10-50 IU/l } \\
\text { G-glutamyl transferase normal: F< }<55\end{array}$ & $284 \cdot 1$ & $9-1480$ \\
$\quad$ M <65 IU/l & $93 \cdot 5$ & $17-314$ \\
\hline
\end{tabular}

\section{Results}

The staining patterns were similar to those reported previously ${ }^{7}$ namely: (1) Type 1 pattern (Fig 1). Granular staining of the cytoplasm with perinuclear accentuation and filaments surrounding the neutrophils. This pattern has been found to be specific to primary sclerosing cholangitis patients. ${ }^{7}$ (2) Type 2 pattern (Fig 2). Diffuse cytoplasmic staining with few or no granules and no filaments surrounding the neutrophils. (3) Negative (Fig 3).

The results are shown in Table III. The type 1 pattern of staining was seen in eight of 10 primary sclerosing cholangitis and three of eight chronic active hepatitis patients. No other condition, including normal subjects, showed this pattern of staining.

Our previous study did not identify any type 2 pattern of staining in primary sclerosing cholangitis. ${ }^{7}$ In this study, however one primary sclerosing cholangitis patient showed this pattern of staining. She was five years old and her liver histology was stage 2 showing mild portal and periportal fibrosis. The primary sclerosing cholangitis patient who was negative had a stage 3 liver histology. She was six years old. Both patients were on prednisolone and azathioprine. Interestingly enough, neither patients had an associated inflammatory bowel disease. Their liver biochemical tests were not significantly different from the other patients. There is probably no relationship between disease activity of primary sclerosing cholangitis and the presence of type 1 pattern of staining, although disease activity in this group of patients is difficult to assess as all of them were on some form of treatment.

All three patients with chronic active hepatitis who showed type 1 (primary sclerosing cholangitis) pattern of staining have type 1 chronic active hepatitis. They had no symptoms of chronic liver disease at time of blood sampling but were on prednisolone and azathioprine. One patient's liver histology showed cirrhosis, the other showed mild to moderate chronic active hepatitis with mild lobular component while the remaining patient showed only piecemeal necrosis. Two of these patients had normal sigmoidoscopy and rectal biopsy. One of them had failed endoscopic retrograde cholangiography while the other patient's cholangiography was normal. The remaining patient had undetermined colitis and pyoderma gangronesum. Her cholangiography was entirely normal.

Sixteen patients with non-primary sclerosing cholangitis liver diseases showed type 2 staining. This pattern of staining is non-specific and is not

\begin{tabular}{lccc} 
TABLE III Pattern of staining in different diseases \\
\hline \multicolumn{3}{c}{ Positive } \\
& Type 1 & Type 2 & Negative \\
& & & \\
\hline $\begin{array}{l}\text { Primary sclerosing cholangitis } \\
(\mathrm{n}=10)\end{array}$ & 8 & 1 & 1 \\
$\begin{array}{l}\text { Chronic active hepatitis }(\mathrm{n}=8) \\
\text { Ulcerative colitis }(\mathrm{n}=11)\end{array}$ & 3 & 5 & 0 \\
$\begin{array}{l}\text { Alpha-1 anti-trypsin deficiency } \\
(\mathrm{n}=10)\end{array}$ & 0 & 2 & 9 \\
$\begin{array}{l}\text { Extrahepatic bile duct atresia } \\
(\mathrm{n}=12)\end{array}$ & 0 & 7 & 3 \\
$\begin{array}{l}\text { Normal }(\mathrm{n}=21) \\
\text { Total }(\mathrm{n}=72)\end{array}$ & 0 & 4 & 8 \\
\hline
\end{tabular}




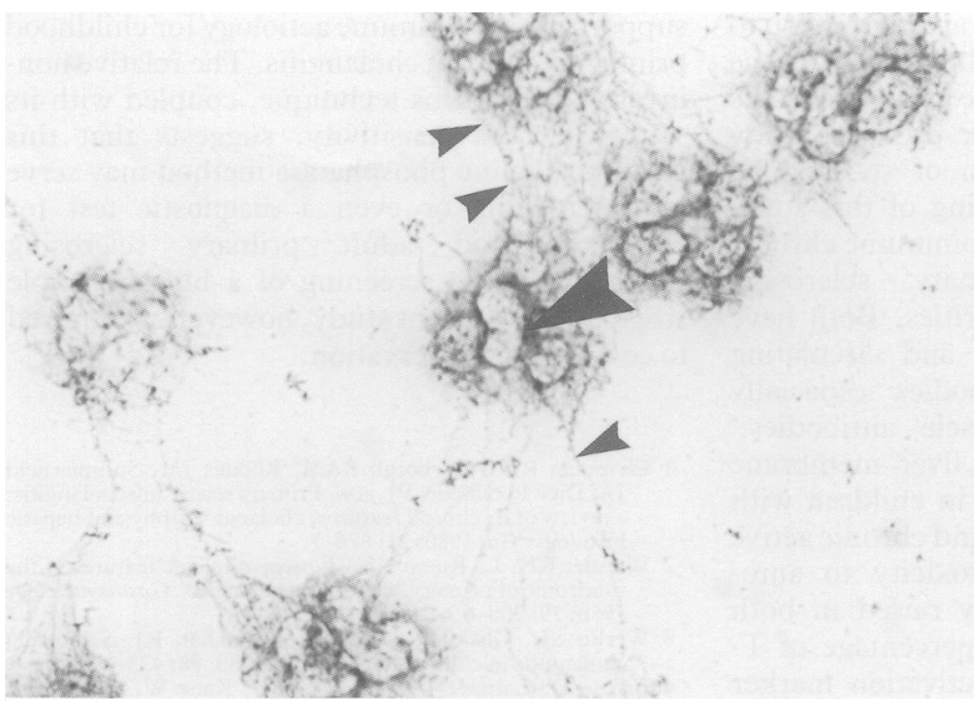

Figure 1: Type 1 pattern of staining. This shows filaments around the neutrophils (small arrows) and the granular cytoplasm with perinuclear accentuation (big arrow).

Figure 2: Type 2 pattern. Note the diffuse cytoplasmic staining with no filaments.

Figure 3: Normal subjects do not show any staining. The outline of the cell is marked by small arrows. The nucleus is indicated by the big arrow. considered as diagnostic for primary sclerosing cholangitis.

\section{Discussion}

The presence of an antineutrophil antibody in adult primary sclerosing cholangitis has been described by several groups. ${ }^{7-10}$ Duerr $e t a l^{9}$ has reported (using an enzyme linked immunosorbent assay followed by indirect immunofluorescence method) that the finding of a combination of raised immunoglobulin $G$ neutrophil antibody and perinuclear immunofluorescence staining was $65 \%$ sensitive and $100 \%$ specific for primary sclerosing cholangitis compared with other chronic liver diseases. Other workers have not found such high specificity, however. ${ }^{10}$ Sharma et al found that the sensitivity was only $43 \%$ and the antibody was detected in primary biliary cirrhosis as well as other chronic liver diseases. ${ }^{10} \mathrm{We}$ have previously described an alternative approach for detection of antineutrophil antibody in adult primary sclerosing cholangitis. ${ }^{7}$ This test uses immunoalkaline phosphatase and identified a type 1 pattern which, in patients with chronic liver diseases, is highly specific for primary sclerosing cholangitis.

This present study confirms both the sensitivity and specificity of this test in childhood primary sclerosing cholangitis (specificity and sensitivity were $95 \%$ and $80 \%$ respectively). Because of this, the nature of the antigenic target is of interest especially in relationship to pathogenesis. The antigen is proteinaceous and heat stable to at least $37^{\circ} \mathrm{C}$ for one hour. ${ }^{7}$ Despite intensive efforts, the exact nature of the antigen remains unknown. It, however, definitely differs from the Wegener's granulomatosis - that is, not serine proteinase 3 , and it is not a myeloperoxidase or cathepsin G. ${ }^{79} \mathrm{Klein}$ et $a l$ has reported the identification of a $60 \mathrm{KD}$ epitope of the neutrophil which was specific for primary sclerosing cholangitis using western blot." This has not been confirmed, however, by this laboratory.?

In view of the invasive nature of endoscopic retrograde cholangiography, the high specificity and sensitivity as shown in this paper suggest further investigation of the antineutrophil antibody assay as a screening or a diagnostic test in children. The relatively small number of patients, however, and the presence of type 1 pattern in three of eight chronic active hepatitis patients indicate that investigation of more patients will be required to verify this test before it can truly be accepted as diagnostically useful.

Unlike the antineutrophil antibody in Wegener's granulomatosis, the antineutrophil antibody titre in adult primary sclerosing cholangitis does not correlate with the clinical activity, biochemistry or liver histology of the patients. ${ }^{7}$ It is likely that the situation in children is similar (see result). It is, therefore, possible that the antibody represents an epiphenomenon and has no pathogenic significance. The numbers are too small to determine whether the antibody is specific for primary sclerosing cholangitis, as opposed to primary sclerosing cholangitis with ulcerative colitis. It may be important, however, as a prognostic marker for those ulcerative colitis 
patients who have the antibody and may go on to develop primary sclerosing cholangitis in the future. ${ }^{7}$ It is of interest that, one of three chronic active hepatitis patients in our previous study also showed a type 1 pattern of staining, ${ }^{7}$ a similar proportion to the finding of this study (three of eight). Childhood autoimmune chronic active hepatitis and primary sclerosing cholangitis share many similarities. Both have raised concentrations of IgG and circulating non-organ specific autoantibodies especially antinuclear and smooth muscle antibodies. Furthermore, antibodies to a liver membrane protein preparation are found in children with primary sclerosing cholangitis and chronic active hepatitis. ${ }^{12}$ Lymphocyte cytotoxicity to autologous hepatocytes is similarly raised in both conditions. ${ }^{12}$ In addition, the percentage of $\mathrm{T}$ lymphocytes expressing the activation marker HLA-DR is similarly increased in primary sclerosing cholangitis and chronic active hepatitis. The finding of a shared specific antineutrophil antibody in both primary sclerosing cholangitis and chronic active hepatitis thus further suggests the possibility that chronic active hepatitis and primary sclerosing cholangitis may represent a spectrum of disease rather than two completely different diseases.

In comparison with adult patients, where the type 1 pattern was seen in $30 \%$ ulcerative colitis patients, ${ }^{7}$ no such pattern was seen in any of the paediatric ulcerative colitis patients. While this difference could have been because of sample size, alternatively, paediatric ulcerative colitis may differ pathogenetically from adult ulcerative colitis.

In conclusion the finding of a specific antineutrophil antibody associated with both adult and childhood primary sclerosing cholangitis cases suggests that both groups may share a same mechanism in their pathogenesis. It also further supports an autoimmune aetiology for childhood primary sclerosing cholangitis. The relative noninvasiveness of this technique, coupled with its specificity and sensitivity, suggests that this immunoalkaline phosphatase method may serve as a screening or even a diagnostic test for childhood and adult primary sclerosing cholangitis. The screening of a bigger sample size than this present study, however, is required to confirm this observation.

1 Chapman RWG, Arborgh BAM, Rhodes JM, Summerfield JA, Dick R, Scheuer PJ, et al. Primary sclerosing cholangitis: a review of its clinical features, cholangiography and hepatic histology. Gut 1980; 21: 870-7.

2 Wiesner RH, La Russo NF. Clinicopathologic features of the syndrome of primary sclerosing cholangitis. Gastroenterology 980; 79: 200-6

3 Werlin SL, Glicklich M, Jona J, Starshak RJ. Sclerosing cholangitis in childhood. $\mathcal{F}$ Pediatr 1980; 96: 433-5.

4 Freese D, Latimer JS, Gilberstadt S, Kane W, Sharp HL Sclerosing cholangitis associated with inflammatory bowe disease. Clin Pediatr 1982; 21: 11-6.

5 Spivak W, Grand RJ, Eraklis A. A case of primary sclerosing cholangitis. Gastroentrology 1982; 82: 129-32.

6 El-Shabrawi M, Wilkinson ML, Portmann B, Mieli-Vergani G, Chong SK, Williams R, Mowat AP. Primary sclerosing cholangitis in childhood. Gastroenterology 1987; 92: 122635 .

7 Lo SK, Fleming KA, Chapman RW. Prevalence of antineutrophil antibody in primary sclerosing cholangitis and ulcerative colitis using an alkaline phosphatase method. Gut 1992; 33: 1370-5

8 Snook JA, Chapman RW, Fleming K, Jewell DP. Antineutrophil nuclear antibody in ulcerative colitis. Crohn's disease and primary sclerosing cholangitis. Clin Exp Immunol 1989; 76: 30-3.

9 Duerr RH, Targan SR, Landers CI, LaRusso NF, Lindsay $\mathrm{KL}$, Wiesner RH, et al. Neutrophil Cytoplasmic antibodies: A link between primary sclerosing cholangitis and ulcerative colitis. Gastroenterology 1991; 100: 1385-91.

10 Sharma AN, Rabson AR, Kaplan MM. Perinuclear antineutrophil cytoplasmic antibodies in primary sclerosing neutrophil cytoplasmic antibodies in

11 Klein R, Eisenburg J, Weber P, Seibold F, Berg PA Significance and specificity of antibodies to neutrophils detected by western blotting for the serological diagnosis of primary sclerosing cholangitis. Hepatology 1991; 14: 114752

12 Mieli-Vergani G, Lobo-Yeo A, McFarlane BM, McFarlane IG, Mowat AP, Vergani D. Different immune mechanism leading to autoimmunity in primary sclerosing cholangitis and autoimmune chronic active hepatitis of childhood. Hepatology 1989; 9: 198-203. 